\title{
A Genetic Polymorphism in GCKR may be Associated with Low High-Density Lipoprotein Cholesterol Phenotype among Filipinos: A Case-control Study
}

\author{
Rody G. Sy, MD, ${ }^{1}$ Jose B. Nevado, Jr., MD, ${ }^{2}$ Eddieson M. Gonzales, MD, ${ }^{1}$ Adrian John P. Bejarin, MD, ${ }^{2,3}$ \\ Aimee Yvonne Criselle L. Aman, MD, ${ }^{2,3}$ Elmer Jasper B. Llanes, MD, ${ }^{1}$ Jose Donato A. Magno, MD, ${ }^{1}$ \\ Deborah Ignacia D. Ona, MD, ${ }^{1}$ Felix Eduardo R. Punzalan, MD, ${ }^{1}$ Paul Ferdinand M. Reganit, MD, ${ }^{1}$ \\ Lourdes Ella G. Santos, MD, ${ }^{1}$ Richard Henry P. Tiongco II, MD, ${ }^{1}$ Jaime Alfonso M. Aherrera, MD, ${ }^{1}$ \\ Lauro L. Abrahan IV, MD, ${ }^{1}$ Charlene F. Agustin, $\mathrm{MD}^{1}$ and Eva Maria C. Cutiongco-de la Paz, MD ${ }^{2,3}$

\footnotetext{
${ }^{1}$ Department of Medicine, Philippine General Hospital, University of the Philippines Manila

${ }^{2}$ Institute of Human Genetics, National Institutes of Health, University of the Philippines Manila ${ }^{3}$ Philippine Genome Center, University of the Philippines Diliman, Quezon City
}

\begin{abstract}
Background. Low levels of high-density lipoprotein cholesterol (HDL-c) is a well-recognized risk factor in the development of cardiovascular diseases. Associated gene variants for low HDL-c have already been demonstrated in various populations. Such associations have yet to be established among Filipinos who reportedly have a much higher prevalence of low HDL-c levels compared to other races.

Objective. To determine the association of selected genetic variants and clinical factors with low HDL-c phenotype in Filipinos.

Methods. An age- and sex-matched case-control study was conducted among adult Filipino participants with serum HDL-c concentration less than $35 \mathrm{mg} / \mathrm{dL}(\mathrm{n}=61)$ and those with HDL-c levels of more than $40 \mathrm{mg} / \mathrm{dL}(\mathrm{n}=116)$. Genotyping was done using DNA obtained from blood samples. Candidate variants were correlated with the low HDL-c phenotype using chi-squared test and conditional logistic regression analysis.

Results. Twelve single nucleotide polymorphisms (SNPs) were associated with low HDL-c phenotype among Filipinos with univariate regression analysis. The variant rs1260326 of glucokinase regulator (GCKR) (CT genotype: adjusted $\mathrm{OR}=5.17$; $\mathrm{p}$-value=0.007; TT genotype: adjusted $\mathrm{OR}=6.28$; $\mathrm{p}$-value=0.027) remained associated with low HDL-c phenotype, together with hypertension and elevated body mass index, after multiple regression analysis.

Conclusion. The variant rs1260326 near GCKR is associated with low HDL-c phenotype among Filipinos. Its role in the expression of low HDL-c phenotype should be further investigated prior to the development of possible clinical applications.
\end{abstract}

Keywords: cardiovascular disease, dyslipidemia, GCKR, genetics, single nucleotide polymorphism, rs1260326

Paper presented in the 49th Annual Convention and Scientific Meeting, Philippine Heart Association, "Pharmacogenetics and Cardiovascular Care: The Future and Now" on May 25, 2018, at EDSA Shangri-la Hotel, Mandaluyong, Philippines.

Paper presented in the $67^{\text {th }}$ Joint AHA and ACC Annual Scientific Session and Expo, American College of Cardiology, "ASCVD Risk in Southeast Asia: Genetic Susceptibility to Cardiovascular Disease Among Filipinos", on March 11, 2018, at Orlando, Florida.

Corresponding author: Rody G. SY, MD

Section of Cardiology

Department of Medicine

Philippine General Hospital, University of the Philippines Manila

Taft Avenue, Ermita, Manila 1000, Philippines

Email: rodysymd@gmail.com / rgsy@up.edu.ph 


\section{INTRODUCTION}

High-density lipoprotein cholesterol (HDL-c) is a strong and independent inverse predictor of cardiovascular disease. In the Lipid Research Clinics Coronary Primary Prevention Trial (LRC-CPPT) ${ }^{1}$ and the Framingham Heart Study, ${ }^{2}$ high HDL-c levels were associated with decreased risk and incidence of coronary heart disease. On the other hand, the Prospective Cardiovascular Münster (PROCAM) study, $^{3}$ the Israel Ischemic Heart Disease Study, ${ }^{4}$ and Prospective Epidemiological Study of Myocardial Infarction (PRIME), ${ }^{5}$ observed increased cardiovascular risk and mortality associated with low HDL-c levels.

Compared to other populations, Filipinos have a higher prevalence of low HDL-c levels. This could potentially explain why diseases of the cardiovascular system remain top causes of mortality in the Philippines, with $12.7 \%$ of total deaths due to ischemic heart diseases and $9.8 \%$ due to cerebrovascular disease. ${ }^{6}$ According to the National Nutrition and Health Survey conducted in 2013, ${ }^{7}$ the prevalence of low HDL-c was 71.3\%, affecting 7 out of 10 Filipino adults. In comparison, lower prevalence of low HDL-c was noted among Hispanics (64.1\%), non-Hispanic Blacks (40.5\%), non-Hispanic Whites (46.9\%) and Northwestern Chinese (60.9\%). ${ }^{8,9}$ These differences in low HDL-c prevalence across ethnicities may be explained by variations in genetic profiles. In addition, Filipinos are underrepresented in previous genetic studies. Knowledge of unique Filipino phenotypes for low HDL-c phenotypes may aid in addressing cardiovascular disease in the Philippines.

Several genetic variants have been associated with HDL-c metabolism in some ethnic groups. In a study involving Sorbs, a Slavic minority in Germany, it was shown that a THO complex 5 (THOC5) polymorphism affects HDL-c levels through the regulation of genes involved in HDL-c synthesis such as apolipoprotein $A 1$ (APOA1), scavenger receptor class $B$ member 1 (SCARB1), and $A T P$ binding cassette transporter $G 8(A B C G 8) .{ }^{10}$ In another study involving Arabs, variants in cholesteryl ester transfer protein (CETP) and LIM and cysteine-rich domains protein 1 (LMCD1) were associated with low HDL-c. ${ }^{11}$ Among Hispanics and specifically among Mexicans, a variant in ATP-binding cassette transporter $A 1$ (ABCA1) was implicated. ${ }^{12,13}$

However, these findings cannot be generalized across populations due to inter-ethnic variability in genetic profiles. A population-specific study is needed to yield relevant observations to explain unique phenotypes. Therefore, this study aimed to investigate the presence of associated genetic variants and assess the contribution of significant clinical factors with low HDL-c phenotype among Filipinos.

\section{MATERIALS AND METHODS}

\section{Study design}

We conducted an age- and sex-matched case-control study, with low HDL-c cases defined as participants with at least two documented low HDL-c determinations (either a combination of at least one reading of below $35 \mathrm{mg} / \mathrm{dL}$ and one reading below $40 \mathrm{mg} / \mathrm{dL}$ or two readings below $35 \mathrm{mg} /$ dL). Controls were defined as those with two documented HDL-c readings of more than $40 \mathrm{mg} / \mathrm{dL}$.

\section{Study Size}

Sample size was computed using the recessive model to provide the largest sample size estimate, a minimum minor allele frequency of $20 \%$ (MAF $\geq 0.20$ ), and an alpha of 0.05 , with a case-to-control ratio of $1: 2$. The computed minimum sample size was set at 62 cases and 124 controls.

\section{Setting and enrollment of participants}

Adult participants were recruited from the ambulatory clinics of the Philippine General Hospital as well as various communities and medical facilities within Metro Manila from July 2013 to March 2017. Such recruitment sites were considered by virtue of the volume as well as variety of patients seen.

\section{Eligibility criteria}

The following criteria were used to enroll participants: (a) aged 18 years or older, (b) of Filipino descent up to the $3^{\text {rd }}$ degree of consanguinity, and (c) able to provide independent consent. Participants were excluded if they had any of the following conditions at the time of enrollment: decompensated heart failure, decompensated chronic lung disease, decompensated chronic liver disease, end-stage renal disease, active malignancy, secondary hypertension, secondary dyslipidemia, pregnancy, and $3^{\text {rd }}$ degree of consanguinity within enrolled participants.

Two controls were recruited for every case among individuals from the study population who had normal or elevated HDL-c levels. Controls were individually matched to cases by age and sex. Cases and controls were defined according to the criteria mentioned earlier.

\section{Clinical data collection}

Demographic and clinical characteristics of the participants were obtained through interview and chart review. Lipid profile levels were measured and recorded.

\section{Customization of genotyping chip}

We used a customized GoldenGate Genotyping (GGGT) beadchip (Illumina, Inc., San Diego, CA, USA), designed in 2012 using candidate single nucleotide polymorphisms (SNPs) from both coding and noncoding regions, including intergenic and intronic SNPs, which have shown evidence of association with lipid transport and 
Table 1. Clinical characteristics of study participants

\begin{tabular}{|c|c|c|c|}
\hline Characteristics & $\begin{array}{l}\% \text { Participants with } \\
\text { low HDL-c }(n=61)\end{array}$ & $\begin{array}{c}\% \text { Participants with } \\
\text { normal HDL-c }(n=116)\end{array}$ & $p$-value ${ }^{*}$ \\
\hline Age in years, mean (SD) & $50(10)$ & $52(10)$ & 0.38 \\
\hline Males, $\%$ & 86.9 & 87.9 & 0.84 \\
\hline HDL-c in mg/dL, mean (SD) & $33.05(7.00)$ & $53.64(11.76)$ & $<0.001^{\dagger}$ \\
\hline \multicolumn{4}{|l|}{ Comorbidities, \% } \\
\hline Elevated BMI (>25 kg/m²) & 71.4 & 35.7 & $<0.001^{\dagger}$ \\
\hline Hypertension & 86.9 & 31.0 & $<0.001^{\dagger}$ \\
\hline Diabetes & 42.6 & 1.7 & $<0.001^{\dagger}$ \\
\hline Reduced eGFR (<60 mml/min) & 4.9 & 4.3 & 0.80 \\
\hline \multicolumn{4}{|l|}{ Lifestyle factors, $\%$} \\
\hline Smoking & 47.5 & 40.5 & 0.33 \\
\hline Alcohol drinking & 78.7 & 69.0 & 0.13 \\
\hline
\end{tabular}

*The following were used to obtain the $p$-values (significance set at $p<0.05$ ): Student's t-test for age and HDL-c, chisquare test for sex, and conditional logistic regression for comorbidities and lifestyle factors. ${ }^{\dagger}$ statistically significant. HDL-c, high-density lipoprotein; BMI, body mass index; eGFR, estimated glomerular filtration rate

metabolism (Table 1). The following databases were used for extensive search of SNPs: PharmGKB (Pharmacogenomics Knowledgebase) database, NHGRI GWAS Catalog (National Human Genome Research Institute Genome-Wide Association Study), PubMED and patent databases (e.g., Patentscope and Espacenet), where risk and protective odds ratios (OR) were provided. The selected SNPs were scored by Illumina Inc. to determine their suitability to discriminate genetic variants, and to estimate their specificity.

\section{DNA extraction and quantification}

We extracted DNA using the QiaAmp DNA Minikit (QIAGEN GmbH, Germany), following the spin protocol for blood buffy coat specified in the manufacturer's instruction manual. DNA was quantified using a spectrometer at 260 $\mathrm{nm}$ and stored at $-20^{\circ} \mathrm{C}$ until use. All DNA samples had absorbance ratio $\left(\mathrm{A}_{260} / \mathrm{A}_{280}\right)$ above 1.80 ; this is a measure of quality of the sample.

\section{Genotyping}

Customized genotyping of candidate SNPs was performed using DNA microarray technology following the GoldenGate Genotyping (GGGT) assay protocol specified in the manufacturer's manual. After microarray processing, the beadchip was imaged on the HiScan System and data from these images were analyzed using Genotyping module v.1.9.4 (GenomeStudio software version 2011.1, Illumina Inc, USA). Variant selection and genotyping methods were similar to methods done in earlier studies. ${ }^{14-15}$

\section{Data analyses}

\section{Quality control}

To ensure high quality data, GenomeStudio and PLINK version 2.05.10 16 were used to identify and remove participants and markers that may lead to false-positive and false-negative associations. We included Genotype data from participants with call rates $>95 \%$ after evaluation with GenomeStudio, and with individual missingness $<0.05$ (missingness test) in PLINK. The following thresholds were used for the genotype data: minor allele frequency of 0.01 (frequency test), genotype missingness of 0.05 (missingness test), and Hardy-Weinberg Equilibrium (HWE) of controls of $\mathrm{p}<0.001$ (HWE test). A test for epistasis was also done to account for interaction between variants.

\section{Determination of genotypic effect}

We performed chi-square tests to assess significant differences among variants of interest, namely, allelic and genotypic association tests (additive, dominant, and recessive models). These models were identified based on the distribution of genotypes. Fisher-Irwin exact tests for allelic and genotypic associations were done, setting the cut-off at Bonferroni-corrected $\alpha$, if applicable.

\section{Logistic regression analysis}

Upon determination of models, we recoded genotypes according to the significant model for univariate analysis in Stata 13 (College Station, Texas). Univariate logistic regression analysis was done to determine association of SNPs with low HDL-c phenotype, with cut-off $\mathrm{p}$-value set at 0.05. Multiple logistic regression was performed to assess contribution of associated clinical factors. Quality control and statistical analyses were similar to the methods done in studies published earlier. ${ }^{14-15}$

\section{Ethics statement}

The study protocol was approved by the University of the Philippines Manila - Research Ethics Board. All participants were required to provide written informed consent. Approval was sought from administrators or authorities of the areas where participants were enrolled. 


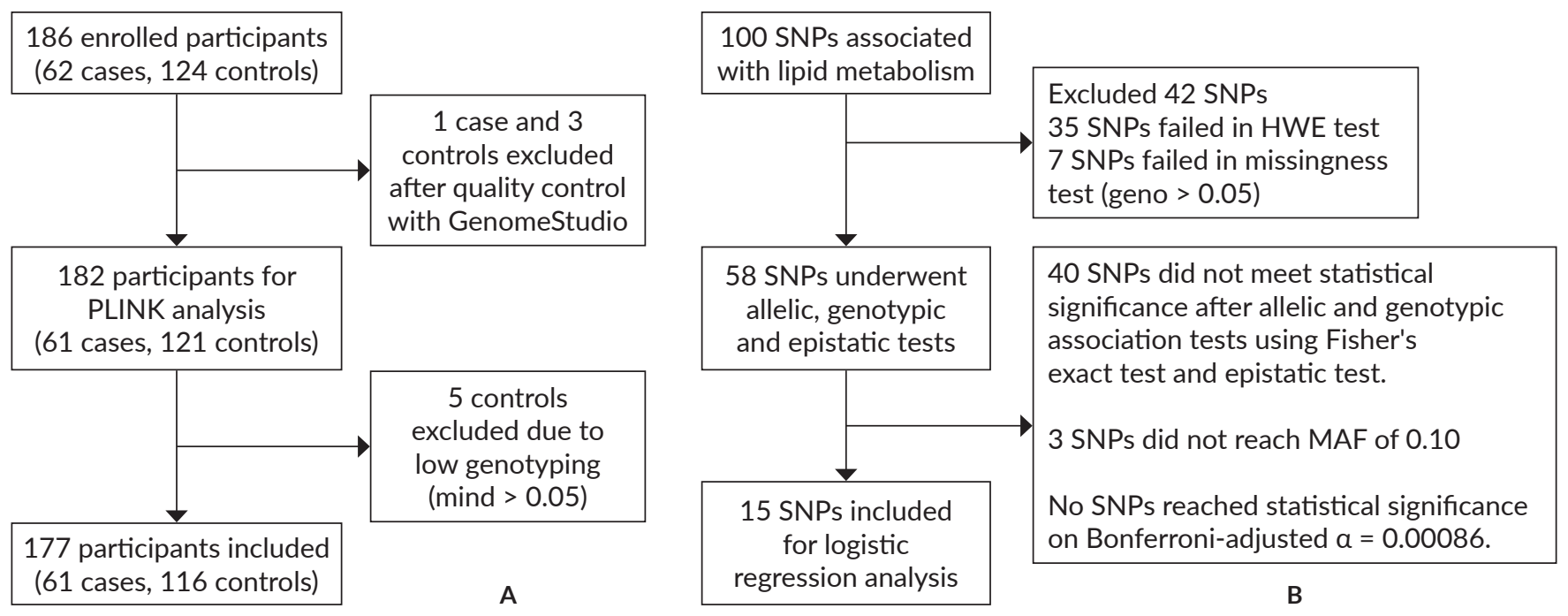

Figure 1. Overview of data processing and analysis. A total of 182 participants (A) and 58 SNPs (B) were analyzed to determine association of clinical metadata and genetic variants with low HDL.

Abbreviations: mind, individual missingness; SNP, single nucleotide polymorphism; HWE, Hardy-Weinberg equilibrium; geno, genotypic missingness; MAF, minor allele frequency

\section{RESULTS}

A total of 186 participants were initially recruited for the study, with 62 low HDL-c participants (cases) and 124 normal HDL-c participants (controls). After quality control, 177 participants (61 cases and 116 controls) remained (Figure 1A).

Among the initial list of 100 SNPs known to be associated with HDL-c metabolism and transport, 42 SNPs were excluded after quality control using Hardy-Weinberg exact test, individual missingness test, and minor allele frequency threshold as criteria. Thirty-eight (38) SNPs were excluded after failing to meet statistical significance on genotypic association analysis using Fisher's test (Bonferroni-adjusted $\alpha=0.0008)$, and 2 were removed after checking for epistasis. Three (3) SNPs have MAF $>0.10$. Fifteen (15) SNPs were included in the univariate regression analysis (Figure 1B).

More participants in the low HDL-c group have elevated BMI (71.4\%), hypertension (86.9\%) and diabetes (42.6\%). Participants with low HDL-c levels are expected to have higher frequency of the said conditions since these comorbidities are known cardiovascular risk factors. The average HDL-c measurement among cases was $33.05 \mathrm{mg} /$ $\mathrm{dL}$, a much lower value than the controls at $53.64 \mathrm{mg} / \mathrm{dL}$ $(\mathrm{p}<0.001)$. (Table 1)

Univariate analysis was used to determine the association of the remaining fifteen (15) SNPs with low HDL-c levels. Of these, 12 SNPs (7 SNPs with additive, 3 with dominant, and 2 with recessive genotypic effects) showed statistically significant association $(\mathrm{p}<0.005)$ while exhibiting crude ORs above 2.5 after crude analysis (Table 2). This implies their clinical significance for potential application in management of cardiovascular diseases once verified in a Filipino cohort.
Among the twelve variants listed in Table 2, rs662799 in $A P O A 5$ had the highest crude OR of 27.88 with a p-value of 0.002 . Three variants, namely rs673548, rs6754295 and rs676210, are located in the same locus and are commonly associated with $A P O B$.

Multiple regression analysis was conducted to account for the effect of clinical variables on the association of the genetic variants with low HDL-c (Table 3). Variable selection was done to select the variant most statistically associated with the low HDL-c phenotype. The variant rs1260326 retained its association with low HDL-c phenotype when adjusted for the presence of hypertension and elevated BMI (Table 3).

Type 2 diabetes mellitus (T2DM) is also known to be associated with low HDL-c. In this study, we did post-hoc matching for T2DM and attempted to include the variable into the equation. Of the 28 participants with T2DM, 26 were shown to have low HDL-c. T2DM conferred 41 times higher odds of having low HDL-c on exact logistic regression analysis (95\% CI 9.58, 376.75; $\mathrm{p}<0.0001$ ). Adjusting for T2DM on conditional logistic regression analysis showed increased odds ratios for both additive and dominant model (Table 3). However, it was observed that the confidence intervals widened. This may be due to the difference in distribution of the participants with and without T2DM (Table 4), and the conditional regression model used for matched samples.

A post-hoc subgroup analysis was done to observe the association of the SNP with low HDL-c separately among those with T2DM and those without the T2DM (Table 4). The exact logistic regression analysis done to check association among participants with T2DM may have been limited by the small sample size; however, it was observed 
Table 2. Significant SNPs after univariate analysis

\begin{tabular}{|c|c|c|c|c|}
\hline SNP & Nearest Gene & Genotype & Crude OR (95\% Cl) & $p$-value ${ }^{*}$ \\
\hline \multirow[t]{2}{*}{ rs1260326 } & GCKR & CT vs. CC & $3.01(1.44,6.29)$ & $0.003^{\dagger}$ \\
\hline & & TT vs. CC & $3.96(1.27,12.35)$ & 0.018 \\
\hline \multirow[t]{2}{*}{ rs1532624 } & CETP & $A C$ vs. $A A$ & $2.13(0.71,6.42)$ & 0.179 \\
\hline & & CC vs. AA & $6.29(2.24,17.70)$ & $<0.001^{\dagger}$ \\
\hline \multirow[t]{2}{*}{ rs662799 } & APOA5 & CT vs. CC & $2.88(1.22,6.76)$ & $0.015^{\dagger}$ \\
\hline & & TT vs. CC & $27.88(3.24,239.50)$ & $0.002^{\dagger}$ \\
\hline \multirow[t]{2}{*}{ rs673548 } & $A P O B$ & AG vs. GG & $6.61(2.48,17.60)$ & $<0.001^{\dagger}$ \\
\hline & & AA vs. GG & $11.58(3.68,36.39)$ & $<0.001^{\dagger}$ \\
\hline \multirow[t]{2}{*}{ rs6754295 } & $A P O B$ & GT vs. TT & $4.47(1.94,10.27)$ & $<0.001^{\dagger}$ \\
\hline & & GG vs. TT & $8.69(3.14,24.06)$ & $<0.001^{\dagger}$ \\
\hline \multirow[t]{2}{*}{ rs676210 } & $A P O B$ & AG vs. GG & $4.04(1.65,9.88)$ & $0.002^{\dagger}$ \\
\hline & & AA vs. GG & $7.00(2.51,19.54)$ & $<0.001^{\dagger}$ \\
\hline \multirow[t]{2}{*}{ rs9733352 } & DOCK1 & CG vs. GG & $2.22(0.92,5.35)$ & 0.076 \\
\hline & & CC vs. GG & $3.96(1.70,9.26)$ & $0.001^{\dagger}$ \\
\hline rs2144300 & GALNT2 & TC/TT vs CC & $3.18(1.62,6.24)$ & $0.001^{\dagger}$ \\
\hline rs2231142 & $A B C G 2$ & $\mathrm{AC} / \mathrm{CC}$ vs $\mathrm{AA}$ & $3.76(1.75,8.06)$ & $0.001^{\dagger}$ \\
\hline rs255049 & DPEP3 & TT vs CT/TT & $5.94(2.26,15.62)$ & $<0.001^{\dagger}$ \\
\hline$r s 285^{\ddagger}$ & $L P L$ & TC/TT vs CC & $3.45(1.71,6.95)$ & $0.001^{\dagger}$ \\
\hline rs $7240405^{\ddagger}$ & LIPG/SMUG1P1 & GG vs AG/AA & $3.69(1.72,7.94)$ & $0.001^{\dagger}$ \\
\hline
\end{tabular}

*significance set at $p<0.005$ using conditional logistic regression

tstatistically significant

¥not included in the multiple regression analysis due to missing genotypes

SNP, single nucleotide polymorphism; Chr, chromosome; OR, odds ratio, $\mathrm{Cl}$, confidence interval; GCKR, glucokinase regulator; CETP, cholesteryl ester transfer protein; APOA5, apolipoprotein A5; APOB, apolipoprotein B; DOCK1, dedicator of cytokinesis protein 1; GALNT2, N-acetylgalactosaminyl-transferase 2; ABCG2, ATP binding cassette subfamily G member 2; DPEP3, dipeptidase 3; LPL, lipoprotein lipase; LIPG, lipase G, endothelial type; SMUG1P1, single-strand-selective monofunctional uracil-DNA glycosylase 1 pseudogene 1.

Table 3. Different models of association of rs1260326 with low HDL-c among Filipinos

\begin{tabular}{lcccc}
\multicolumn{2}{c}{ Model for rs1260326 } & Crude OR (95\% CI) & Adjusted OR (95\% CI)* & Adjusted OR (95\% Cl) ${ }^{\dagger}$ \\
\multirow{2}{*}{ Additive } & CT vs. CC & $3.01(1.44,6.29)$ & $4.37(1.34,14.22)$ & $23.07(1.49,357.56)$ \\
& TT vs. CC & $3.96(1.27,12.35)$ & $4.68(0.80,27.24)$ & $7.63(0.49,119.50)$ \\
\hline Dominant & TT/CT vs CC & $2.29(1.36,3.83)$ & $2.74(1.24,6.07)$ & $3.59(1.09,11.82)$ \\
\hline
\end{tabular}

*adjusted for hypertension and elevated BMI $\left(\geq 25 \mathrm{~kg} / \mathrm{m}^{2}\right)$; significance set at $p>0.05$ using multiple conditional logistic regression

tadjusted for hypertension, elevated BMI $\left(\geq 25 \mathrm{~kg} / \mathrm{m}^{2}\right)$ and type 2 diabetes mellitus; significance set at $p<0.05$ using multiple conditional logistic regression

$\mathrm{OR}$, odds ratio; $\mathrm{Cl}$, confidence interval

Table 4. Distribution of T2DM among those with normal and low HDL-c

\section{Low HDL-c ( $\mathrm{n}=61) \quad$ Normal HDL-c $(\mathrm{n}=116) \quad$ Total $(\mathrm{N}=177)$}

\begin{tabular}{lccc} 
With T2DM, \% (n) & $42.62(26)$ & $1.72(2)$ & $15.82(28)$ \\
Without T2DM, \% (n) & $57.38(35)$ & $98.28(114)$ & $84.18(149)$ \\
\hline
\end{tabular}

that all diabetic participants with the risk allele $\mathrm{T}$ had low HDL-c. Among participants without T2DM, both the additive and dominant models of genotypic effect showed increased odds of having low HDL-c among those with the risk allele T. These show that the association of the SNP with low HDL-c may be independent of an individual's T2DM status.

\section{DISCUSSION}

Twelve variants located in $A P O A 5 / B, N$-acetylgalactosaminyl-transferase 2 (GALNT2), ATP binding cassette subfamily $G$ member 2 (ABCG2), dipeptidase 3 (DPEP3), cholesteryl ester transfer protein (CETP), dedicator of cytokinesis protein 1 (DOCK1), and glucokinase regulator (GCKR) are found to 
be associated with low HDL-c phenotype in the Filipino cohort in this study. One genetic variant, rs1260326 in $G C K R$, retained its statistically significant association when adjusted for the presence of hypertension and elevated BMI.

The GCKR gene, located in chromosome 2p, codes for glucokinase regulator protein (GKRP) that is predominantly involved in glucose metabolism in the liver. GKRP modulates the activity of glucokinase, an enzyme that phosphorylates glucose during the first step of glycolysis and glycogen synthesis. GKRP binds glucokinase and relocates it from the cytoplasm to the nucleus where it is held inactive. ${ }^{17}$ Failure in GKRP modulation could result to enhanced glycolysis and increased de novo lipogenesis in the liver. ${ }^{18}$ Variations in GCKR have been reported to be associated with dyslipidemia and changes in metabolic profile. ${ }^{19-21}$

A missense variant in the isomerase domain of GCKR, rs1260326, likely results to conformational changes in GKRP affecting binding to glucokinase. Impaired binding may result in uninhibited glycolysis, which may increase lipogenesis in the liver, especially of triglyceride (TG). Studies by Chasman et al., ${ }^{13}$ Teslovich et al., ${ }^{22}$ and Weissglas-Volkov et al. ${ }^{23}$ showed that the variant was significantly associated with high TG levels. A review by Welty mentioned that higher TG levels facilitate more CETP-mediated transfer resulting in a higher concentration of TG-rich HDL-c. ${ }^{24}$ Compared to cholesteryl ester-rich TG, TG-rich HDL-c is more rapidly metabolized, leading to lower levels of HDL-c. A previous study by Zhou et al. among Chinese patients has already implicated the variant with low HDL-c levels. ${ }^{21}$ However, Varbo and colleagues showed in a cross-sectional study that the SNP is minimally associated with low HDL-c, with note of a stepwise decrease as the number of $\mathrm{T}$ alleles increase $(\sim 0.01 \mathrm{mmol} / \mathrm{L} ; \mathrm{p}=0.02) .{ }^{25}$ Among Filipinos, those who have the TT or TC genotype are likely to have 3.59 times higher odds of having low HDL-c levels than those with the $\mathrm{CC}$ genotype.

The risk allele ( $\mathrm{T}$ allele) frequency of rs 1260326 among the study controls was $18 \%$, which is similar to that in South Asians (20\%), higher than Africans (9\%), and lower than admixed Americans (36\%), Europeans (41\%), East Asians $(48 \%){ }^{26}$

Knowing the role of GCKR in glucose metabolism, the presence of concomitant diabetes mellitues as a possible confounder was tested. Although a larger sample size for subgroup analyses among participants with diabetes and those without diabetes is recommended, the current analysis showed a trend that the association of rs1260326 and low HDL-c persists, regardless of DM status. For subsequent studies, it may be prudent to recommend that T2DM status (with or without T2DM), as well as control of T2DM be included as a priori variables for analysis.

The clinical factors found to be associated with low HDL-c phenotype in this study population are hypertension and elevated BMI. These are typical features of metabolic syndrome, which is a cluster of risk factors for cardiovascular disease. ${ }^{27}$ The group of Liu et al. ${ }^{28}$ has characterized the link between low HDL-c and other components of metabolic syndrome. They found that both men and women with initially low HDL-c develop hypertension as the most common additional and new-onset metabolic syndrome component. They purported that low HDL-c concentration is connected to other components of the metabolic syndrome, mainly as a consequence of systemic low-grade inflammation and APOA1 dysfunction. The association between hypertension and HDL-c levels is further substantiated in their opposing effects in the number of circulating endothelial progenitor cells in the vascular endothelium. ${ }^{29}$ The link between elevated BMI and low HDL-c can be understood in terms of insulin resistance resulting from adipose tissue resistant to the antilipolytic effects of insulin leading to increased hepatic flux of fatty acids from dietary sources and intravascular lipolysis. ${ }^{30}$

This is a hypothesis-generating study and larger followup prospective studies are needed to confirm current findings. In addition, the identified SNPs in this study that were not previously associated with low HDL-c in other populations should be interpreted with caution. Generalizing results and applying them to Filipinos will only be recommended upon replication and validation of positive associations among these SNPs.

\section{CONCLUSION}

The frequency of the variant rs1260326 in GCKR, adjusted for the presence of hypertension and elevated BMI, is significantly associated with low HDL-c phenotype of Filipinos, Further study is recommended to assess the role of the variant in the expression of low HDL-c phenotype among Filipinos. This will provide insight on the prevention and clinical management of cardiovascular diseases.

\section{Acknowledgments}

The authors thank Professors Cynthia Cordero and Kim Cochon for their assistance in statistical analysis, as well as all the laboratory personnel, research staff, and coordinators of the Institute of Human Genetics, National Institutes of Health, University of the Philippines, Manila and the Philippine Genome Center whose valuable contributions made this project possible.

This study was approved and funded by the Philippine Council for Health Research and Development of the Department of Science and Technology through the Grants in Aid (GIA) Program.

\section{Statement of Authorship}

For conceptualization and methodology: RG Sy, EC Cutiongco-de la Paz,JB Nevado, FR Punzalan, EB Llanes, JA Magno, DD Ona, PM Reganit, LG Santos, and RP Tiongco.

For funding acquisition, project administration and supervision: RG Sy, EC Cutiongco-de la Paz, JB Nevado, and AL Aman. 
For investigation: RG Sy, EC Cutiongco-de la Paz, JB Nevado, FR Punzalan, EB Llanes, JA Magno, DD Ona,

PM Reganit, LG Santos, RP Tiongco, JM Aherrera, LL Abrahan, CF Agustin, AL Aman, and AP Bejarin.

For data curation and formal analysis: JB Nevado, AL Aman, and AP Bejarin.

Interpretation of data: RG Sy, EC Cutiongco-de la Paz, JB Nevado, FR Punzalan, EB Llanes, JA Magno, DD Ona, PM Reganit, LG Santos, RP Tiongco, JM Aherrera, LL Abrahan, CF Agustin, AL Aman, and AP Bejarin.

Writing of original draft and visualization: JB Nevado, AL Aman, AP Bejarin, and EM Gonzales.

Revising the manuscript for significant intellectual content: RG Sy, EC Cutiongco-de la Paz, and JB Nevado,.

All authors approved the final version of the manuscript to be published.

\section{Author Disclosure}

The following authors have no conflicts of interest to declare: Dr. Abrahan, Dr. Agustin, Dr. Aman, Dr. Bejarin, Dr. Cutiongco-de la Paz, Dr. Magno, Dr. Nevado, Dr. Punzalan, and Dr. Reganit.

Dr. Aherrera: Received honoraria for lectures for Servier, Astrazeneca, GX International.

Dr. Llanes: Received honoraria for lectures for Servier, MSD, Bayer, Astrazeneca, Boehringer Ingelheim, Menarini, Novartis and LRI-Therapharma.

Dr. Ona: Received speaker grants from Astrazeneca, Servier, Viatris, Sanofi and Zydus; Advisory Board Member of Sanofi.

Dr. Santos: Past speaker for the following: Servier, Astrazeneca, MSD, LRI-Therapharma, OEP, Zuellig, Boehringer Ingelheim, Pascual Labs, Kanarb, Menarini, Ajanta, Torrent; Advisory Board Member of Zuellig and Kanarb.

Dr. Sy: Received research grants from MSD, Novartis, Pfizer and Servier; received honoraria for lectures for Astrazeneca, Corbridge, MSD, Novartis, Servier, LRITherapharma and Zuellig-Amgen; Advisory Board Member of Astrazeneca, MSD, Novartis, Pfizer, Servier and ZuelligAmgen.

Dr. Tiongco: Past speaker for CME for ADP Pharma for levamlodipine molecule and 2019 CME module development for levamlodipine besylate with ADP Pharma. Past resource speaker on clopidogrel for Sanofi, and made a module for clopidogrel for Getz. Prepared module for best/ limited use for inclusion in clinical practice guidelines.

\section{Funding Source}

This paper and the project were funded by the Philippine Council on Health Research and Development of the Department of Science and Technology.

\section{REFERENCES}

1. Rifkind BM. Lipid research clinics coronary primary prevention trial: Results and Implications. Am J Cardiol. 1984 Aug; 54(5):30-4.

2. Wilson PW, Abbott RD, Castelli WP. High-density lipoprotein cholesterol and mortality. The Framingham Heart Study. Arteriosclerosis. 1988 Nov-Dec; 8(6):737-41.

3. Assmann G, Schulte H. The Prospective Cardiovascular Münster (PROCAM) study: prevalence of hyperlipidemia in persons with hypertension and/or diabetes mellitus and the relationship to coronary heart disease. Am Heart J. 1988 Dec; 116(6):1713-24.

4. Goldbourt U, Yaari S. Cholesterol and coronary heart disease mortality. A 23-year follow-up study of 9902 men in Israel. Arteriosclerosis. 1990 Jul-Aug; 10(4):512-9.

5. Luc G, Bard JM, Ferrieres J, Evans A, Amouyel P, Arveiler D, et al. Value of HDL-c cholesterol, apolipoprotein AI, lipoprotein AI, and lipoprotein AI/A-II in prediction of coronary heart disease: the PRIME study. Arterioscler Thromb Vasc Biol. 2002; 22(7):1155-61.

6. Philippine Statistics Authority. Death in the Philippines, 2016 [Internet]. 2018 [cited 2018 May]. Available from: https://psa.gov. $\mathrm{ph} /$ content/deaths-philippines-2016\#: :text=Reported\%20deaths\% 20 in $\% 202016 \% 20$ reached,of\%201\%2C591\%20persons\%20died\% 20daily.

7. Food and Nutrition Research Institute - Department of Science and Technology (FNRI-DOST). Philippine Nutrition Facts and Figures 2013: Clinical and Health Survey [PDF]. 2015. Available from: http:// enutrition.fnri.dost.gov.ph/site/preview.php? $\mathrm{xx}=\% 20$ uploads/2013 FaF_Clinical_and_Health_Survey.pdf

8. Willey JZ, Rodriguez CJ, Carlino RF, Moon YP, Paik MC, BodenAlbala B, et al. Race-ethnic differences in the association between lipid profile components and risk of myocardial infarction: The Northern Manhattan Study. Am Heart J. 2011 May; 161(5):886-92.

9. Ge P, Dong C, Ren X, Weiderpass E, Zhang C, Fan H, et al. The high prevalence of low HDL-c-cholesterol levels and dyslipidemia in rural populations in northwestern China. PLoS One. 2015 Dec; 10(12): $\mathrm{e} 0144104$.

10. Keller M, Schleinitz D, Förster J, Tönjes A, Böttcher Y,FischerRosinskyet A, al.THOC5: a novel gene involved in HDL-c-cholesterol metabolism. J Lipid Res. 2013 Nov; 54(11):3170-6.

11. Wakil SM, Ram R, Muiya NP, Andres E, Mazhar N, Hagos S, et al. A common variant association study reveals novel susceptibility loci for low HDL-c-cholesterol levels in ethnic Arabs. Clin Genet. 2016; 90(6):518-25.

12. Klarin D, Damrauer SM, Cho K, Sun YV, Teslovich TM, Honerlaw J, et al. Genetics of blood lipids among 300,000 multi-ethnic participants of the Million Veteran Program. Nat Genet. 2018 Nov; 50(11):1514.

13. Weissglas-Volkov D, Aguilar-Salinas CA, Nikkola E, Deere KA, CruzBautista I, Arellano-Campos O, et al. Genomic study in Mexicans identifies a new locus for triglycerides and refines European lipid loci. J Med Genet. 2013 May; 50(5):298-308.

14. Reganit PFM, Nevado JB, Cutiongco-De La Paz EMC, Taquiso JL, Aman AYCL, Llanes EJB, Magno JDA, Ona DID, Punzalan FER, Santos LEG, Tiongco RHP, Aherrera JM, Abrahan LL, Agustin CF, Bejarin AJP, Sy RG. 2020. BAG6 variant rs805303 is nominally associated with ACEi-induced cough among Filipinos. Philipp J Sci 149(1): 35-41.

15. Sy RG, Nevado JB, Llanes EJB, Magno JDA, Ona DID, Punzalan FER, Reganit PFM, Santos LEG, Tiongco RHP 2nd, Aherrera JAM, Abrahan LL 4th, Agustin CF, Aman AYCL, Bejarin AJP, Cutiongco-De La Paz EMC. 2020. The Klotho Variant rs36217263 Is Associated with Poor Response to Cardioselective Beta-Blocker Therapy among Filipinos. Clin Pharmacol Ther 107(1): 221-226.

16. Purcell S, Neale B, Todd-Brown K, Thomas L, Ferreira MA, Bender D, Maller J, Sklar P, De Bakker PI, Daly MJ, Sham PC. PLINK: a tool set for whole-genome association and population-based linkage analyses. The American journal of human genetics. 2007 Sep 1;81(3):559-75. http://pngu.mgh.harvard.edu/purcell/ plink 
17. Raimondo A, Rees MG, Gloyn AL. Glucokinase regulatory protein: complexity at the crossroads of triglyceride and glucose metabolism. Curr Opin Lipidol. 2015 Apr; 26(2):88.

18. Santoro N, Caprio S, Pierpont B, Van Name M, Savoye M, Parks EJ. Hepatic de novo lipogenesis in obese youth is modulated by a common variant in the GCKR gene. J Clin Endocr Metab 2015 Aug; 100(8):E1125-32.

19. Mohás M, Kisfali P, Járomi L, Maász A, Fehér E, Csöngei V, et al. GCKR gene functional variants in type 2 diabetes and metabolic syndrome: Do the rare variants associate with increased carotid intimamedia thickness? Cardiovasc Diabetol. 2010 Nov; 9:79.

20. Simons N, Dekker JM, van Greevenbroek MM, Nijpels G, 't Hart LM, van der Kallen CJ, et al. A common gene variant in glucokinase regulatory protein interacts with glucose metabolism on diabetic dyslipidemia: The combined CODAM and Hoorn studies. Diabetes Care. 2016 Oct; 39(10):1811-7.

21. Zhou YJ, Hong SC, Yin RX, Yang Q, Cao XL, Chen WX. Polymorphisms in the GCKR are associated with serum lipid traits, the risk of coronary artery disease and ischemic stroke. Int J Clin Exp Med. 2015 Jul; 8(7):10678.

22. Chasman DI, Paré G, Zee RY, Parker AN, Cook NR, Buring JE, et al. Genetic loci associated with plasma concentration of lowdensity lipoprotein cholesterol, high-density lipoprotein cholesterol, triglycerides, apolipoprotein A1, and Apolipoprotein B among 6382 white women in genome-wide analysis with replication. Circ Cardiovasc Genet. 2008; 1(1):21-30.

23. Teslovich TM, Musunuru K, Smith AV, Edmondson AC, Stylianou IA, Koseki M, et al. Biological, clinical and population relevance of 95 loci for blood lipids. Nature. 2010 Aug; 466(7307):707.
24. Welty FK. How do elevated triglycerides and low HDL-c-cholesterol affect inflammation and atherothrombosis? Curr Cardiol Rep. 2013 Sep; 15(9):400.

25. Varbo A, Benn M, Tybjærg-Hansen A, Grande P, Nordestgaard BG. TRIB1 and GCKR polymorphisms, lipid levels, and risk of ischemic heart disease in the general population. Arterioscler Thromb Vasc Biol. 2011; 31(2):451-7. doi: 10.1161/ATVBAHA.

26. 1000 Genomes Project Consortium. A global reference for human genetic variation. Nature. 2015 Oct; 526(7571):68.

27. Alberti KG, Eckel RH, Grundy SM, Zimmet PZ, Cleeman JI, Donato KA, et al. Harmonizing the metabolic syndrome: a joint interim statement of the international diabetes federation task force on epidemiology and prevention; national heart, lung, and blood institute; American heart association; world heart federation; international atherosclerosis society; and international association for the study of obesity. Circulation. 2009 Oct; 120(16):1640-5.

28. Liu X, Tao L, Cao K, Wang Z, Chen D, Guo J, et al. Association of high-density lipoprotein with development of metabolic syndrome components: a five-year follow-up in adults. BMC Public Health. 2015; 15(1):412.

29. Shimizu Y, Sato S, Koyamatsu J, Yamanashi H, Nagayoshi M, Kadota $\mathrm{K}$, et al. Association between high-density lipoprotein-cholesterol and hypertension in relation to circulating CD34-positive cell levels. J Physiol Anthropol. 2017;36(1):26.

30. Klop B, Elte J, Cabezas M. Dyslipidemia in obesity: mechanisms and potential targets. Nutrients. $2013 \mathrm{Apr}$; 5(4):1218-40. 